\title{
QUALIDADE DE GAMETAS COM A TÉCNICA DE ENDOSCOPIA EM CURIMBA (PROCHILODUS LINEATUS)
}

\author{
Estefânia de Souza Andrade¹, Mônica Rodrigues Ferreira', Daniella Aparecida de \\ Jesus Paula1, Luis David Solis Murgas¹, Viviane de Oliveira Felizardo¹, Galileu \\ Crovatto Veras ${ }^{1}$ \\ 1 UFLA \\ Correspondência: Estefânia Andrade: esandrade@bol.com.br
}

\begin{abstract}
RESUMO: A endoscopia pode facilitar o manejo em piscicultura e possibilita a realização da sexagem de forma precisa, mesmo que seu uso ainda seja restrito. A curimba é uma espécie de peixe reofílica, utilizada em experimentos como espécie modelo para peixes migradores. Este trabalho teve como objetivo viabilizar a utilização do endoscópio rígido na sexagem de reprodutores de curimba e determinar se a técnica altera os índices reprodutivos. Foram examinados 16 curimbas, nos quais foi identificado o sexo através da introdução do endoscópio pelo poro urogenital para visualização das gônadas. O endoscópio rígido utilizado (modelo Multi-Purpose Rigid ${ }^{\mathrm{TM}}$, Karl Storz, Germany) apresentava ângulo de $30^{\circ}$, com $2,7 \mathrm{~mm}$ de diâmetro e $18 \mathrm{~cm}$ de comprimento. Foi realizada a indução hormonal 20 dias após a aplicação da técnica, as fêmeas receberam duas doses de extrato bruto de hipófise de carpa (EBHC) de 0,4 e 4,0 mg/kg de peixe, com intervalo de 12 horas e os machos receberam uma única dose de $4,0 \mathrm{mg} / \mathrm{kg}$ de peixe de EBHC, no momento da segunda aplicação nas fêmeas. Após 12 horas, procedeu-se a extrusão dos gametas, sendo retirada uma amostra de sêmen para avaliação da morfologia, taxa (\%) e duração (segundos) da motilidade e concentração espermática. Para as fêmeas realizou-se a avaliação do peso da desova e coleta de uma amostra dos ovócitos para avaliar a posição da vesícula germinativa e o diâmetro dos ovócitos. Pode-se observar que $100 \%$ dos machos espermiaram e $62 \%$ das fêmeas desovaram. Além disso, todos os índices reprodutivos foram considerados dentro dos padrões esperados para a espécie. Conclui-se que a endoscopia pode ser utilizada de maneira eficiente na sexagem e na avaliação do grau de maturação gonadal, antes da piracema, sem alterar a eficiência reprodutiva dos peixes.
\end{abstract}

Palavras-chave: endoscópio; peixe; reprodução

\section{QUALITY OF GAMETES WITH ENDOSCOPY TECHNIQUE IN CURIMBA (PROCHILODUS LINEATUS)}

\begin{abstract}
Endoscopy can facilitate for fish and allows the performance of sexed accurately, even if its use is still restricted. The curimba is a rheophilic species of fish, used in experiments as a model species for migratory fish. This study aimed to enable the use of the endoscope in sexing breeding curimba and determine whether the technique alters the reproductive rates. Curimbas 16 were examined, in which sex was identified by the introduction of the pore urogenital endoscope for viewing the gonads. The endoscope used (model Multi-Purpose Rigid TM, Karl Storz, Germany) had a $30^{\circ}$ angle with $2.7 \mathrm{~mm}$ diameter and $18 \mathrm{~cm}$ in length. Hormonal induction was performed 20 days after application of the technique, the females received two doses of crude extract of carp pituitary (CECP) of 0.4 and $4.0 \mathrm{mg} / \mathrm{kg}$ of fish, with an interval of 12 hours and the males receiving a single dose of $4.0 \mathrm{mg} / \mathrm{kg}$ of fish CECP, when the second application in females. After 12 hours, we proceeded to the extrusion of the gametes, which was removed a semen sample for evaluation of morphology, rate (\%) and duration (seconds) motility and sperm concentration. For the females took place evaluating the weight of spawning and collecting a sample of oocytes to evaluate the position of the germinal vesicle and oocyte diameter. It can be observed that $100 \%$ of males and $62 \%$ of female spawned. In addition, all reproductive indices were considered within the standards expected for the species. It follows that the endoscope can be used efficiently in sexing and evaluating the degree of maturation prior to spawning season, without changing the reproductive efficiency of Fish.
\end{abstract}

Key Words: endoscope; fish; reproduction 


\section{INTRODUÇÃO}

Quando mantidos em cativeiro, os peixes reofílicos necessitam da utilização de hormônios para que ocorra a espermiação e desova, visto que os gametas são produzidos naturalmente, porém não há atuação hormonal suficiente para promover a liberação dos gametas sem que haja a indução hormonal. $O$ sucesso da indução hormonal depende tanto da capacidade de diferenciação sexual dos reprodutores como da determinação exata do estádio de desenvolvimento gonadal e, consequentemente, do momento de aplicação dos hormônios (Mylonas et al., 2010).

Atualmente, o processo de seleção de reprodutores é realizado a partir de características macroscópicas, sendo observados em fêmeas o volume e flacidez ventral, bem como o tamanho e a coloração avermelhada da papila urogenital. Nos machos, a seleção é feita exercendo leve pressão abdominal no sentido crânio-caudal, observando-se a existência da espermiação (Woynarovich e Horváth, 1983).

Porém, as características citadas acima podem ser subjetivas, não expressando de forma correta o verdadeiro estado reprodutivo ou sexo dos animais (Lima et al., 2011). Para a sexagem de Salvelinus alpinus, os animais são eutanasiados, por não apresentarem dimorfismo sexual (Mayer et al., 1992). Em salmonídeos as características morfológicas externas não são eficientes para determinação do estado avançado de desenvolvimento gonadal (Martin et al., 1995).

Algumas técnicas podem ser empregadas na avaliação do sexo e estádio de desenvolvimento gonadal. Em esturjões é feita a anestesia do peixe, sendo realizada uma incisão no abdômen e posterior avaliação endoscópica para inspeção das gônadas. Após o exame, a incisão é suturada (Hurvitz et al., 2007). Ainda, segundo estes autores, é possível realizar uma biópsia para avaliação histológica das gônadas.

Em esturjões e surubins a maturação gonadal tem sido avaliada utilizando o endoscópio, através do poro urogenital. Esta técnica tem se mostrado eficaz, e tem como vantagem o fato de não ser invasiva (Divers et al., 2009; Hernandez-Divers et al., 2004; Kynard e Kieffer, 2002). Comparando-se a eficiência entre o ultrassom e a endoscopia, foi constatada que quanto mais invasiva a técnica, maior será a facilidade de visualização, contribuindo, assim na determinação das características reprodutivas dos peixes (Wildhaber et al., 2005).

A ausência de dimorfismo sexual em várias espécies de peixes faz com que a sexagem e a definição da maturação gonadal se tornem tarefas complicadas, sendo necessária a adoção de uma técnica confiável e prática. A curimba, também conhecida como curimbatá, é uma espécie do gênero Prochilodus, que realiza migração entre novembro e janeiro (Pereira et al., 2009). Assim, o objetivo deste trabalho foi avaliar a utilização do endoscópio rígido, via papila urogenital, para sexagem de reprodutores de curimba, bem como determinar se a técnica altera os índices reprodutivos.

\section{MATERIAL E MÉTODOS}

Foram utilizados dezesseis curimbas provenientes da Estação de Piscicultura da Universidade Federal de Lavras, durante o mês de fevereiro de 2010. Os reprodutores foram capturados dos viveiros de alvenaria de $80 \mathrm{~m}^{2}$, com auxílio de redes de arrasto. Em seguida foi realizada a sexagem dos animais através da introdução de um endoscópio rígido (modelo Multi-Purpose Rigid ${ }^{\mathrm{TM}}$, 
Karl Storz, Germany) pelo poro urogenital para visualização das gônadas, com ângulo de $30^{\circ} ; 2,7 \mathrm{~mm}$ de diâmetro e $18 \mathrm{~cm}$ de comprimento, acoplado a uma camisa endoscópica de $5 \mathrm{~mm}$ para insuflação de soro fisiológico estéril, além de cabo de fibra ótica e fonte de luz alógena de 150 Watts que acompanha o endoscópio (Lima et al., 2011).

Para visualização das imagens no computador, foi acoplado ao endoscópio uma micro câmera (MedCam Clinic, Toronto) ligada a uma placa de captura de imagens (Dazzle TM, Pinnacle, United Kingdon).

A técnica consistiu em identificar o sexo do animal, sendo caracterizados como fêmeas quando eram visualizados ovócitos e como machos os que não apresentavam os mesmos. Após este procedimento, os peixes foram medidos, pesados e marcados para a determinação da dose hormonal a ser aplicada. Foi realizada indução hormonal 20 dias após a aplicação da técnica de endoscopia, sendo utilizadas para as fêmeas duas doses de extrato bruto de hipófise de carpa (EBHC), que consistiram de injeções intramusculares de 0,4 e $4,0 \mathrm{mg} / \mathrm{kg}$ de peixe, respectivamente, com intervalo de 12 horas. Já os machos receberam uma única dose de $4,0 \mathrm{mg} / \mathrm{kg}$ de peixe de EBHC, no momento da segunda aplicação nas fêmeas.

Os peixes foram retirados dos tanques 12 horas após a segunda dose do hormônio, a extrusão dos gametas foi realizada através de uma massagem na região ventral no sentido crâniocaudal. O sêmen de cada reprodutor foi acondicionado em tubo de ensaio estéril graduado para medição do volume e os ovócitos foram depositados em béqueres estéreis, sendo posteriormente identificados.

Uma alíquota de $10 \mu \mathrm{L}$ de sêmen in natura de cada animal foi colocada em uma lâmina histológica de vidro e, a seguir, homogeneizada com $40 \mu \mathrm{L}$ de água destilada para determinação da taxa (\%) e duração (segundos) da motilidade espermática. A duração da motilidade foi considerada desde a ativação até que apenas $10 \%$ das células espermáticas apresentassem motilidade. A concentração espermática foi calculada com o auxílio da câmara de Neubauer no microscópio óptico, sendo o sêmen diluído em solução de formol citrato na proporção de 1:1.000 (sêmen:solução diluente) (Felizardo et al., 2010).

Para análise da morfologia espermática, o sêmen foi diluído em formol citrato na proporção de 1:1.000 (sêmen: solução diluente) e em seguida realizado um esfregaço por amostra e levado ao microscópio para leitura em aumento de $1000 \mathrm{X}$. Para cada amostra 100 espermatozóides foram avaliados e o número de células normais e anormais foi expresso em porcentagem.

Para a avaliação do desenvolvimento ovariano, duas amostras de ovócitos foram coletadas, sendo a primeira para realizar a mensuração do diâmetro através da fixação em solução de Gilson $(50 \mathrm{~mL}$ de álcool $60 \%, 440 \mathrm{~mL}$ de água destilada, 7 $\mathrm{mL}$ de ácido nítrico, $10 \mathrm{~g}$ de cloreto mercúrico - $\mathrm{HgCl}_{2}$ e $9 \mathrm{~mL}$ de ácido acético glacial) por 30 minutos. Em seguida, os ovócitos foram medidos (mm) com auxílio de ocular micrométrica (10x), sob microscópio óptico (40x). A segunda amostra foi fixada em líquido de Serra $\left(60 \mathrm{~mL}\right.$ de álcool $90^{\circ} \mathrm{GL} ; 30 \mathrm{~mL}$ de formalina e $10 \mathrm{~mL}$ de ácido acético glacial), para avaliação da migração da vesícula germinativa com auxílio de microscópio estereoscópico.

\section{RESULTADOS E DISCUSSÃO}

Foram identificados oito machos e oito fêmeas, identificadas inicialmente pelos caracteres macroscópicos como abaulamento de ventre e coloração de 
papila urogenital. Os animais possuíam peso médio de 0,418 $\pm 0,113$ e $0,298 \pm 0,840 \mathrm{~kg}$ e comprimento total de $31,0 \pm 5,0$ e $28,59 \pm 3,2 \mathrm{~cm}$, fêmeas e machos, respectivamente.

A endoscopia mostrou-se uma ferramenta eficiente na sexagem em curimba, sendo que os resultados foram confirmados com a reprodução posteriormente, Após a indução hormonal foi verificado que os peixes responderam de maneira eficiente, sendo que $100 \%$ dos machos espermiaram e $62 \%$ das fêmeas desovaram, não havendo uma influência negativa da técnica sobre o processo reprodutivo.

A técnica de endoscopia é utilizada para a identificação do sexo, mesmo fora da época de reprodução ou em peixes imaturos (Bryan et al., 2005; Hurvitz et al., 2007; Kynard e Kieffer, 2002; Swenson et al., 2007). Em Medicina Veterinária ela é utilizada para examinar órgãos internos de peixes via inserção do endoscópio na cavidade celomática (Wildhaber et al., 2005).

As técnicas de manipulação dos reprodutores durante a primeira fase de desenvolvimento dos gametas, constituindo a proliferação, crescimento e diferenciação dos gametas (gametogênese e vitelogênese) podem prejudicar o desempenho reprodutivo, gerando estresse aos reprodutores do plantel e inibindo a segunda fase da reprodução (ovulação e espermiogênese) (Blythe et al., 1994). Além disso, o estresse pode alterar ou inibir a motivação dos peixes de expressarem os comportamentos reprodutivos (Chandroo et al., 2004).

A concentração espermática média dos reprodutores neste trabalho foi de $75,81 \times 10^{9} \quad \pm \quad 35,59 \times 10^{9}$ espermatozóides $/ \mathrm{mL}$. Os valores de referência variam entre $16,8 \times 10^{9}$ e

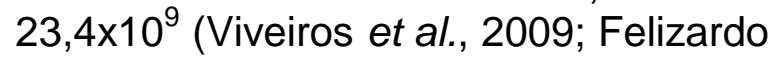
et al., 2010). O aumento da concentração média encontrada, provavelmente se deve à época de indução hormonal, na qual foi realizada a reprodução destes animais, já no final da época reprodutiva. De acordo com Silva et al. (2009), a concentração espermática é correlacionada positivamente com o período que ocorre a indução hormonal, sendo maior no fim da piracema.

A taxa de motilidade encontrada do sêmen de curimba de todos os animais foi de $100 \%$ e a média do volume observado foi de $1,16 \pm 0,38 \mathrm{~mL}$. Os valores médios da duração da motilidade encontrados foram de $139 \pm 102$ segundos, sendo superior aos valores encontrados por Murgas et al. (2007) que relataram de 24 a 88 segundos.

A morfologia espermática apresentou médias de 19,25 $\pm 6,11 \%$ para anormalidades maiores, 7,62 \pm $1,4 \%$ para anormalidades menores e $26,87 \pm 5,79 \%$ para anormalidades totais. As anormalidades maiores são desenvolvidas durante a espermatogênese, devido a fatores externos que podem acometer os reprodutores, como estresse e enfermidades. Anormalidades menores como cabeça isolada $(28,0 \pm 4,2 \%)$ e cauda isolada $(18 \pm 2,8 \%)$ já foram visualizados por Moraes et al. (2004). Porém, estes autores descreveram que os percentuais elevados deste tipo de anormalidades podem estar relacionados ao procedimento de preparação e coleta do sêmen (Streit-Jr et al., 2008).

Cinco fêmeas responderam à indução hormonal, sendo que a média

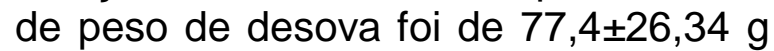
(Tabela 1). A variação de resposta à indução hormonal em fêmeas é variável e depende de uma série de fatores (Pereira et al., 2009). Estes mesmos autores obtiveram $100 \%$ de desova em fêmeas de curimba. Em pirapitinga, foi 
encontrado usando a mesma dosagem hormonal utilizada, porém com hipófise de salmão, um índice de desova de $48 \%$ (Nagahama, 1994).

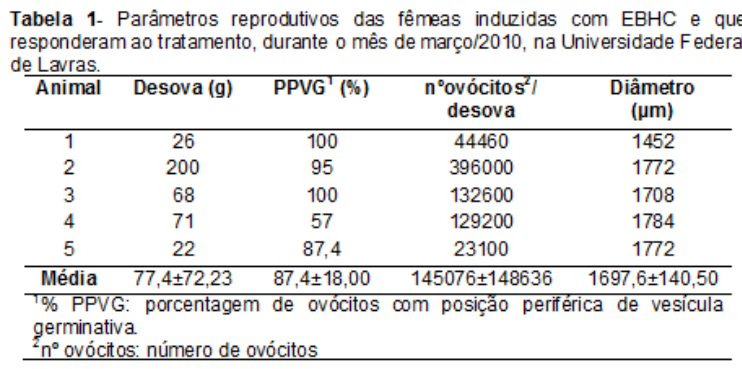

O peso de desova difere de alguns resultados encontrados. Pereira et al. (2009) observaram entre os meses de novembro/2005 e janeiro/2006, peso médio de 221,7 g com dose de 0,5 e 5 $\mathrm{mg} / \mathrm{kg}$ de $\mathrm{EBHC}$ e citam também que a época de reprodução de curimba é de novembro a janeiro. Silva et al. (2009) observaram peso médio de desova em novembro, dezembro e janeiro de $177,5 \pm 96,2 ; 263,7 \pm 135,0 ; 264,4 \pm 142,9$ $\mathrm{g}$, respectivamente, também utilizando doses de 0,5 e $5,0 \mathrm{mg} / \mathrm{kg}$ e concluem que as melhores desovas foram nos meses de dezembro e janeiro. Nossos resultados podem estar relacionados com a época em que o experimento foi realizado.

A média de porcentagem de ovócitos com posição periférica de vesícula germinativa foi de $87,4 \pm 18,0 \%$ (Tabela 1). Esta média não difere dos dados encontrados por Pereira et al. (2009), que foi de $94,0 \%$. A posição periférica da vesícula germinativa indica que os ovócitos estão em final de maturação, contrário do que é encontrado em ovócitos imaturos ou em maturação, em que a posição do núcleo é central (Murgas et al., 2011).

Uma análise dos diâmetros dos ovócitos intraovarianos como indicador do grau de desenvolvimento gonadal das fêmeas foi utilizada pela primeira vez por (Fenerich-Verani et al., 1984) com curimbatá Prochilodus scrofa. O diâmetro médio encontrado neste trabalho foi de 1697,6 $\pm 140,5 \mu \mathrm{m}$ (Tabela 1) e é próximo ao observado por diversos autores.

Foi observado que o sucesso da fertilização ocorreu somente em fêmeas com ovócitos de diâmetros superiores a 735,55 $\mu \mathrm{m}$ e que as fêmeas que apresentavam ovócitos significativamente maiores não respondiam aos tratamentos hormonais estando este resultado de acordo com relatos de Fenerich-Verani et al. (1984). Foi encontrado para curimba um tamanho médio de $2640 \mu \mathrm{m}$ (Hainfellner, 2011), e Pereira et al. (2009) encontrou diâmetro de $1360 \mu \mathrm{m}$.

A sexagem e a avaliação do grau de maturação gonadal em reprodutores de curimba podem melhorar os índices reprodutivos dos cardumes, além de facilitar o manejo nas pisciculturas. Porém, as técnicas invasivas se mal aplicadas podem prejudicar a saúde e o desempenho reprodutivo, gerando estresse aos reprodutores do plantel, introduzindo patógenos e inibindo a ovulação, sendo ineficientes (Blythe et al., 1994). Além disso, o estresse pode alterar ou inibir a motivação dos peixes de expressarem outros comportamentos, como os reprodutivos (Chandroo et al., 2004).

\section{CONCLUSÃO}

A técnica de endoscopia surge como uma possibilidade para avaliação dos reprodutores, sendo não invasiva e de fácil execução. É visto que os reprodutores utilizados neste experimento responderam de maneira esperada à indução hormonal, sem alteração dos parâmetros reprodutivos avaliados. Assim, esta técnica pode ser utilizada de maneira eficiente para sexagem e avaliação de grau de maturação gonadal, antes da piracema, sem alterar a eficiência reprodutiva dos peixes. 


\section{REFERÊNCIAS}

BLYTHE, B.; HELFRICH, L.A.; BEAL, W.E. et al. Determination of sex and maturational status of striped bass (Morone saxatilis) using ultrasonic imaging. Aquaculture, v.125, n.1-2, p.175-184, 1994.

BRYAN, J.L.; WILDHABER, M.L.; NOLTIE, D.B. Examining Neosho madtom reproductive biology using ultrasound and artificial photothermal cycles. North American Journal of Aquaculture, v.67, n.3, p.221-230, 2005.

CHANDROO, K.P.; DUNCAN, I.J.H.; MOCCIA, R.D. Can fish suffer? Perspectives on sentience, pain, fear and stress. Applied Animal

Behaviour Science, v.86, p.225-250, 2004.

DIVERS, S.J.; BOONE, S.S.; HOOVER, J.J. et al. Field endoscopy for identifying gender, reproductive stage and gonadal anomalies in free-ranging sturgeon (Scaphirhynchus) from the lower Mississippi River. Journal of Applied Ichthyology, v.25, p.68-74, 2009.

FELIZARDO, V.O.; MELLO, R.A.; MURGAS, L.D.S. et al. Effect of cryopreservant combinations on the motility and morphology of curimba (Prochilodus lineatus) sperm. Animal Reproduction Science, v.122, n.3-4, p.259263, 2010.

FENERICH-VERANI, N.; GODINHO, H.M.; NARAHARA, M.Y. The size composition of the eggs of curimbata, Prochilodus scrofa Steindachner 1881, induced to spawn with human chorionic gonadotropin (HCG).

Aquaculture, v.42, n.1, p.37-41, 1984.

HAINFELLNER, P. Desenvolvimento ovariano do Prochilodus lineatus em dois sistemas de produção: tanques rede e viveiros escavados. 2011. Jaboticabal, 73f. Dissertação (Mestrado em Aquicultura) - Curso de Pósgraduação em Aquicultura, Centro de Aquicultura, Universidade Estadual Paulista.

HERNANDEZ-DIVERS, S.J.; BAKAL, R.S.; HICKSON, B.H. et al. Endoscopic sex determination and gonadal manipulation in gulf of Mexico sturgeon (Acipenser oxyrinchus desotoi). Journal of Zoo and Wildlife

Medicine, v.35, n.4, p.459-470, 2004.

HURVITZ, A.; JACKSON, K.; DEGANI, G. et al. Use of endoscopy for gender and ovarian stage determinations in Russian sturgeon (Acipenser gueldenstaedtii) grown in aquaculture. Aquaculture, v.270, p.158-166, 2007.

KYNARD, B.; KIEFFER, M. Use of a borescope to determine the sex and egg maturity stage of sturgeons and the effect of borescope use on reproductive structures. Journal of Applied Ichthyology, v.18, n.4-6, p.505-508, 2002.

LIMA, F.S.M.; MURGAS, L.D.S.; FELIZARDO, V.O. et al. Determinação do sexo em catfish de canal (Ictalurus punctatus) utilizando endoscopia. Revista Ceres, v.58, n.3, p.284287, 2011.

MARTIN, S.W.; LONG, J.A.; PEARSONS, T.N. Comparison of Survival, Gonad Development, and Growth between Rainbow Trout with and without Surgically Implanted Dummy Radio Transmitters. North American Journal of Fisheries Management, v.15, n.2, p.494-498, 1995.

MAYER, I.; SCHMITZ, M.; BORG, B. et al. Seasonal endocrine changes in male and female Arctic charr (Salvelinus alpinus). I. Plasma levels of three androgens, 17a-hydroxy-20ßdihydroprogesterone, and $17 \beta$-estradiol.

Canadian Journal of Zoology, v.70, p.37-42, 1992.

MORAES, G.V.; STREIT JR., D.P.; RIBEIRO, R.P. et al. Ação de diferentes indutores reprodutivos hormonais no aparecimento de anormalidades morfológicas em espermatozóides de piavuçu (Leporinus macrocephalus), curimbatá (Prochilodus lineatus) e carpa comum (Cyprinus carpio). Boletim do Instituto de Pesca, v.30, n.2, p.109-116, 2004.

MURGAS, L.D.S.; MILIORINI, A.B.; FREITAS, R.T.F. et al. Criopreservação do sêmen de curimba (Prochilodus lineatus) mediante adição de diferentes diluidores, ativadores e crioprotetores. Revista Brasileira de Zootecnia, v.36, n.2, p.526-531, 2007.

MURGAS, L.D.S.; FELIZARDO, V.O.; FERREIRA, M.R. et al. Importância da avaliação dos parâmetros reprodutivos em peixes nativos. Revista Brasileira de Reprodução Animal, v.35, n.2, p.186-191, 2011.

MYLONAS, C.C.; FOSTIER, A.; ZANUY, S. Broodstock management and hormonal manipulations of fish reproduction. General and comparative endocrinology, v. 165, p.516-534, 2010. 
NAGAHAMA, Y. Endocrine regulation of gametogenesis in fish. International Journal Developmental Biology, v. 38, p. 217-229, 1994.

PEREIRA, G.J.M.; MURGAS, L.D.S.; SILVA, J.M.A. et al. Indução da desova de curimba (Prochilodus lineatus) utilizando eCG E EBHC. Revista Ceres, v.56, n.3, p. 156-160, 2009.

SILVA, J.M.A.; MURGAS, L.D.S.; FELIZARDO, V.O. et al. Características seminais e índices reprodutivos de curimba (Prochilodus lineatus) em diferentes períodos reprodutivos. Revista Brasileira de Saúde e Produção Animal, v.10, n.3, p.668-677, 2009.

STREIT-JR, D.P.; SIROL, R.N.; RIBEIRO, R.P.et al. Qualitative parameters of the piapara semen (Leporinus elongatus Valenciennes, 1850). Brazilian Journal of Biology, v.68, n.2, p. 373-377, 2008.

SWENSON, E.A.; ROSENBERGER, A.E.; HOWELL, P.J. Validation of endoscopy for determination of maturity in small salmonids and sex of mature individuals. Transactions of the American Fisheries Society, v.136, p.994-998, 2007.

VIVEIROS, A.T.M.; ORFÃO, L.H.; MARIA, A.N. et al. A simple, inexpensive and successful freezing method for curimba Prochilodus lineatus (Characiformes) semen. Animal Reproduction Science, v.112, p.293-300, 2009.

WILDHABER, M.L.; PAPOULIAS, D.M.; DELONAY, A.J. et al. Gender identification of shovelnose sturgeon using ultrasonic and endoscopic imagery and the application of the method to the pallid sturgeon. Journal of Fish Biology, v.67, n.1, p.114-132, 2005.

WOYNAROVICH, T.U; HORVÁTH, L. A propagação artificial de peixes de águas tropicais: manual de extensão. Brasília: FAO/CODEVASF/CNPQ, 1983. 220 p. 